\title{
A GENERALIZATION OF $m$-CONVEXITY AND A SANDWICH THEOREM
}

\author{
Teodoro lara, Janusz Matkowski, Nelson Merentes, \\ Roy Quintero, MaŁgorzata Wróbel
}

\begin{abstract}
Functional inequalities generalizing $m$-convexity are considered. A result of a sandwich type is proved. Some applications are indicated.
\end{abstract}

\section{Introduction}

We consider some notions of convexity. To be more detailed assume that $\alpha:[0,1] \rightarrow \mathbb{R}$ is a given function and $I \subset \mathbb{R}$ is an interval such that $t I+\alpha(t) I \subset$ $I$ for all $t \in[0,1]$, where $t I+\alpha(t) I$ denotes the set $\{t x+\alpha(t) y: x, y \in I\}$. In Section 2 we deal with functions satisfying the inequality

$$
f(t x+\alpha(t) y) \leq t f(x)+\alpha(t) f(y)
$$

for all $x, y \in I, t \in[0,1]$, and referred to as a convexity with respect to $\alpha$ (convex wrt $\alpha$ ). It turns out that, under some general conditions on $\alpha$, if $f$ is convex wrt $\alpha$, then $f$ has to be convex; and under a little stronger conditions, $f$ is convex wrt $\alpha$ if and only if it is convex (Proposition 2.1). We note that

Received: 04.10.2016. Accepted: 21.02.2017. Published online: 12.04.2017.

(2010) Mathematics Subject Classification: 26B25, 28A51, 39B62.

Key words and phrases: convexity, convexity with respect to a function, $m$-convexity, functional inequality, functional equation, sandwich theorem, a characterization of $L^{p}$-norm. 
this notion is "closer" to the classical convexity if $\alpha$ is a decreasing involution $\left(\alpha \circ \alpha=\left.\mathrm{id}\right|_{[0,1]}\right)$. It occurs, in particular if, for some $p>0$,

$$
\alpha(t)=\left(1-t^{p}\right)^{1 / p}, \quad t \in[0,1] .
$$

Moreover, given a number $m>0$, we say that $f$ is $m$-convex with respect to an involution $\alpha$, if

$$
f(t x+m \alpha(t) y) \leq t f(x)+m \alpha(t) f(y), \quad x, y \in I, t \in[0,1] .
$$

For $\alpha(t)=1-t, \quad t \in[0,1]$, this notion coincides with the concept of $m$ convexity introduced by Toader [15] 1984 (see also [5, 7, 8, 13]). We compare the $m$-convexity with the convexity with respect to a mean (Aumann [2], 1933).

In Section 3 we deal with $m$-convex functions when $0<m<1$. We note that, in general, the $m$-convex functions do not share the properties of convex ones (Corollary 3.3). However, we show that a function is affine, if it is $m$-affine (Remark 3.4). For every $m \in(0,1)$ we construct a polynomial $h$ of degree 4 such that $f:=\left.h\right|_{[0,+\infty)}$ has the following properties: $f$ is a diffeomorphic $m$-convex self-mapping of $[0,+\infty)$, but not convex in $[0,+\infty)$. It shows that the $m$-convex functions do not have the property that their graphs are placed above the supporting straight-lines. On the other hand, for any sequence $\left(t_{n} \in(0,1): n \in \mathbb{N}\right)$ such that $\lim _{n \rightarrow+\infty} t_{n}=1$ there is a sequence $\left(s_{n} \in(0,1): n \in \mathbb{N}\right)$, with $\lim _{n \rightarrow+\infty} s_{n}=0, t_{n}+s_{n}<1$ for every $n \in \mathbb{N}$, and

$$
f\left(t_{n} x+s_{n} y\right) \leq t_{n} f(x)+s_{n} f(y), \quad x, y \in[0,+\infty), n \in \mathbb{N}
$$

so $m$-convex functions are, to some extent, quite close to convex ones.

In Section 4, assuming that $0<m<1$, we prove the following result of a sandwich type: if $f:(0,+\infty) \rightarrow \mathbb{R}$ is $m$-convex, then there exists a convex function $h: I \rightarrow \mathbb{R}$ such that

$$
f(x) \leq h(x) \leq m f\left(\frac{x}{m}\right), \quad x>0 .
$$

The main result of the last section says that every $m$-convex function $f:(0,+\infty) \rightarrow \mathbb{R}$ such that $\liminf _{x \rightarrow 0+} f(x) \leq 0$, where $m>1$, is a linear function. 


\section{Convexity with respect to a function and $m$-convexity}

Let us begin with the following

Proposition 2.1. Let $\alpha:[0,1] \rightarrow \mathbb{R}$ be a continuous function and $I \subset \mathbb{R}$ be an open nonempty interval such that $t I+\alpha(t) I \subset I$ for all $t \in[0,1]$. Suppose that a function $f: I \rightarrow \mathbb{R}$ is convex with respect to $\alpha$, i.e., $f$ satisfies the inequality

$$
f(t x+\alpha(t) y) \leq t f(x)+\alpha(t) f(y), \quad x, y \in I, t \in[0,1] .
$$

(i) If there exists $t_{0} \in(0,1)$ such that $t_{0}+\alpha\left(t_{0}\right)=1$, then $f$ is convex in the classical sense; moreover, if $0 \in I, f(0) \leq 0$ and $0 \leq t+\alpha(t) \leq 1$ for all $t \in[0,1]$, then $f$ satisfies (2.1) if and only if it is convex.

(ii) If there are $t_{1}, t_{2} \in[0,1]$ such that $t_{1}+\alpha\left(t_{1}\right)<1$ and $t_{2}+\alpha\left(t_{2}\right)>1$, then $(0,+\infty) \subset$ I and $f(x)=f(1) x$ for all $x \in I$.

Proof. (i) By the assumption we have

$$
f\left(t_{0} x+\left(1-t_{0}\right) y\right) \leq t_{0} f(x)+\left(1-t_{0}\right) f(y), \quad x, y \in I,
$$

so $f$ is Jensen convex [4].

Note that there are $x, y \in I, x \neq y$, such that the function $[0,1] \ni t \mapsto$ $t x+\alpha(t) y$ is not constant.

Indeed, in the opposite case, for every pair $(x, y) \in I^{2}, x \neq y$, there would exist a constant $c(x, y)$ such that $t x+\alpha(t) y=c(x, y)$ for all $t \in[0,1]$, whence $y \neq 0$ and

$$
\alpha(t)=\frac{c(x, y)}{y}-\frac{x}{y} t, \quad t \in[0,1] .
$$

Since $\alpha$ does not depend on $x$ and $y$, it follows that $x=y$. This contradiction proves the claim.

Take $x, y \in I, x \neq y$, such that the function $[0,1] \ni t \mapsto t x+\alpha(t) y$ is not constant. Since it is continuous, its range is a nontrivial interval $I(x, y)$. Moreover, applying (2.1) and the Weierstrass Theorem for the continuous function $[0,1] \ni t \mapsto t x+\alpha(t) f(y)$, we get the boundedness from above of $f$ on the interval $I(x, y)$. Now, the Bernstein-Doetsch Theorem (cf. [6, Theorem 6.4.2]) implies that $f$ is convex.

To prove the "moreover" part note first that if $f$ is convex and $f(0) \leq 0$ then $f$ is starshaped, i.e., $f(\lambda x) \leq \lambda f(x)$ for all $\lambda \in[0,1]$ and $x \in I$. Indeed,

$$
f(\lambda x+(1-\lambda) 0) \leq \lambda f(x)+(1-\lambda) f(0) \leq \lambda f(x) .
$$


Hence, for all $x, y \in I, t \in[0,1]$, we get

$$
\begin{aligned}
f(t x+\alpha(t) y) & =f\left(\frac{t}{t+\alpha(t)}(t+\alpha(t)) x+\frac{\alpha(t)}{t+\alpha(t)}(t+\alpha(t)) y\right) \\
& \leq \frac{t}{t+\alpha(t)} f((t+\alpha(t)) x)+\frac{\alpha(t)}{t+\alpha(t)} f((t+\alpha(t)) y) \\
& \leq t f(x)+\alpha(t) f(y) .
\end{aligned}
$$

(ii) By the Darboux property of $\alpha$, between $t_{1}, t_{2}$ there is $t_{0} \in(0,1)$ that $t_{0}+\alpha\left(t_{0}\right)=1$. In view of (i), the function $f$ is convex, so the function $I \ni x \mapsto$ $\frac{f(x)}{x}$ is either monotonic or, for some $x_{0} \in I$, decreasing in $I \cap\left(-\infty, x_{0}\right)$ and increasing in $I \cap\left(x_{0},+\infty\right)$ (see [1] where this "modality" property of convex functions, conjectured by M. Kuczma, has been proved). Since, by (2.1),

$$
\frac{f\left(\left(t_{1}+\alpha\left(t_{1}\right)\right) x\right)}{\left(t_{1}+\alpha\left(t_{1}\right)\right) x} \leq \frac{f(x)}{x}, \quad x \in I
$$

and

$$
\frac{f\left(\left(t_{2}+\alpha\left(t_{2}\right)\right) x\right)}{\left(t_{2}+\alpha\left(t_{2}\right)\right) x} \leq \frac{f(x)}{x}, x \in I,
$$

the function $x \mapsto \frac{f(x)}{x}$ is non-decreasing and non-increasing, so it must be constant.

It follows that in some generalizations of the convexity notion in the form 2.1 it can be reasonable to assume that (see below, Corollary 5.3 .

$$
t+\alpha(t) \leq 1, \quad t \in[0,1] .
$$

Moreover, taking in this proposition $\alpha:[0,1] \rightarrow[0,1]$,

$$
\alpha(t):=1-t, \quad t \in[0,1],
$$

the function $f$ satisfies (2.1) if and only if it is convex. Since in this case we have $(\alpha \circ \alpha)(t)=t$ for all $t \in[0,1]$, it may be sometimes convenient to assume that $\alpha$ is an involution.

We propose the following generalizations of the notion of $m$-convex function introduced by Toader [15]. 
Definition 2.2. Let $\alpha:[0,1] \rightarrow[0,1]$ be a function and $m>0$ be fixed. A subset $X$ of a linear space is said to be convex with respect to $\alpha$ (convex wrt $\alpha$ ), if

$$
x, y \in X \Longrightarrow t x+\alpha(t) y \in X
$$

$m$-convex wrt $\alpha$, if

$$
x, y \in X \Longrightarrow t x+m \alpha(t) y \in X .
$$

We say that a function $f: X \rightarrow \mathbb{R}$ is convex (concave, affine) wrt $\alpha$, if $X$ is convex wrt $\alpha$ and

$$
f(t x+\alpha(t) y) \leq t f(x)+\alpha(t) f(y), \quad x, y \in X, t \in[0,1],
$$

(respectively, if converse inequality or equality holds).

We say that a function $f: X \rightarrow \mathbb{R}$ is $m$-convex ( $m$-concave, $m$-affine) wrt $\alpha$, if $X$ is $m$-convex wrt $\alpha$ and

$$
f(t x+m \alpha(t) y) \leq t f(x)+m \alpha(t) f(y), \quad x, y \in X, t \in[0,1],
$$

(respectively, if converse inequality or equality holds).

Remark 2.3. A function $f: X \rightarrow \mathbb{R}$ is $m$-convex wrt $\alpha$ if and only if its epigraph

$$
E(f):=\{(x, y) \in X \times \mathbb{R}: f(x) \leq y\}
$$

is $m$-convex wrt $\alpha$.

Indeed, assume that $f$ is $m$-convex wrt $\alpha$ and take arbitrary $\left(x_{1}, y_{1}\right)$, $\left(x_{2}, y_{2}\right) \in E(f)$. Then $f\left(x_{1}\right) \leq y_{1}, f\left(x_{2}\right) \leq y_{2}$ and, for arbitrary $t \in[0,1]$,

$$
f\left(t x_{1}+m \alpha(t) x_{2}\right) \leq t f\left(x_{1}\right)+m \alpha(t) f\left(x_{2}\right) \leq t y_{1}+m \alpha(t) y_{2} .
$$

Hence

$$
t\left(x_{1}, y_{1}\right)+m \alpha(t)\left(x_{2}, y_{2}\right)=\left(t x_{1}+m \alpha(t) x_{2}, t y_{1}+m \alpha(t) y_{2}\right) \in E(f),
$$

which shows that the set $E(f)$ is $m$-convex wrt $\alpha$. The converse implication is also easy to verify.

In the sequel we assume that $X=I \subset \mathbb{R}$ is a nonempty interval such that $t I+\alpha(t) I \subset I$ for every $t \in I$, i.e., $I$ is convex wrt $\alpha$ (respectively, $t I+m \alpha(t) I \subset I$ for every $t \in I)$. 
REMARK 2.4. If $\alpha:[0,1] \rightarrow[0,1]$ is a decreasing involution, that is

$$
(\alpha \circ \alpha)(t)=t, \quad t \in[0,1]
$$

then it is a continuous bijection of $[0,1]$. Moreover, replacing $t$ by $\alpha(t)$ in 2.2 , we get

$$
f(\alpha(t) x+m t y) \leq \alpha(t) f(x)+m t f(y), \quad x, y \in I, t \in[0,1]
$$

and repeating this procedure here, we return to 2.2, similarly as in the classical case.

If $\alpha$ is an involution and $m \in(0,1)$ then the interval $I$ must be of the form $[0, b)$ or $(0, b)$ for some $b$ such that $0<b \leq+\infty$.

EXAmple 2.5. For arbitrarily fixed $p>0$, the function $\alpha:[0,1] \rightarrow[0,1]$,

$$
\alpha(t):=\left(1-t^{p}\right)^{1 / p}, \quad t \in[0,1]
$$

is an involution. Moreover,

$$
t+m\left(1-t^{p}\right)^{1 / p} \leq 1, \quad t \in[0,1], \quad p \in(0,1], \quad m \leq 1
$$

For $p=1$ we get $\alpha(t):=1-t(t \in[0,1])$, and the inequality in Definition 2.2 reduces to

$$
f(t x+m(1-t) y) \leq t f(x)+m(1-t) f(y), \quad x, y \in I, t \in[0,1],
$$

which means that the function $f$ is $m$-convex in the sense considered by Toader 15] (see also [5, 7, 16]).

Some generalizations of the classical notion of the convex function are strictly related to the notion of mean.

Let $I \subset \mathbb{R}$ be an interval, and a function $M: I \times I \rightarrow I$ be a mean in $I$, that is

$$
\min (x, y) \leq M(x, y) \leq \max (x, y), \quad x, y \in I .
$$

Clearly, if $J \subset I$ is a subinterval, then $M(J \times J) \subset J$ and $M$ is reflexive, that is

$$
M(x, x)=x, \quad x \in I .
$$


A lot of (already classical) generalizations of the convex function read as follows: A function $f: J \rightarrow I$ is convex (concave, affine) with respect to a mean $M$ in the interval $J$ (Aumann, [2]), if

$$
f(M(x, y)) \leq M(f(x), f(y)), \quad x, y \in J,
$$

(respectively, the opposite inequality, equality) holds.

Note that this definition is correct due to the inclusion $M(J \times J) \subset J$ for every subinterval $J \subset I$, that is equivalent to the mean property of $M$. For $I=\mathbb{R}$ and $M=A$, where $A$ is the arithmetic mean $A(x, y):=\frac{x+y}{2}$, we get the notion of Jensen convex (concave, affine) function in an interval $J \subset \mathbb{R}$; for $I=(0,+\infty)$ and $M=G$, where $G(x, y)=\sqrt{x y}$, we obtain the definition of Jensen geometrically convex function in an interval $J \subset(0,+\infty)$ (cf. for instance [10]).

Remark 2.6. Let $\alpha:[0,1] \rightarrow[0,1]$ be an involution, $m>0$, and an interval $I$ be $m$-convex wrt $\alpha$. For arbitrarily fixed $t \in(0,1)$ such that $\alpha(t) \neq t$, let $N: I^{2} \rightarrow \mathbb{R}$ be given by

$$
N(x, y):=t x+m \alpha(t) y, \quad x, y \in I .
$$

Then

(i) $N$ is a mean in $I$ if and only if $m=1$ and $\alpha(t)=1-t$ (in this case $m$-convexity with respect to $\alpha$ coincides with $m$-convexity);

(ii) if $m<1$ and $N(I \times I) \subset I$, then 0 must belong to the closure of $I$; in particular, if $I \subset[0,+\infty)$, then $I$ must be of the form $[0, b)$ or $(0, b)$ for some $b$ such that $0<b \leq+\infty$.

To see (i) note that, if $N$ is a mean then its reflexivity implies $t+m \alpha(t)=1$. Replacing here $t$ by $\alpha(t)$ and taking into account $\alpha(\alpha(t))=t$ we get $\alpha(t)+$ $m t=1$. These equalities imply that $(1-m)(\alpha(t)-t)=0$, so $m=1$ and, consequently, $\alpha(t)=1-t$. Part (ii) is obvious.

\section{Some properties of $m$-convex functions and an example}

In this section we consider the $m$-convex functions in the sense of Toader [15], that is, we assume in Definition 2.2 that $m<1$ and $\alpha(t)=1-t$ for all $t \in[0,1]$. 
Remark $3.1([15,16])$. Let an interval $I$ be as in Definition 2.2 $(m$-convex wrt $\alpha$ ).

(i) If $m>0$ and $f: I \rightarrow \mathbb{R}$ is $m$-convex, then, for all $x, y, z \in I$,

$$
\begin{aligned}
& x<z<m y \Longrightarrow \frac{f(x)-f(z)}{x-z} \leq \frac{f(z)-m f(y)}{z-m y} ; \\
& m y<z<x \Longrightarrow \frac{f(x)-f(z)}{x-z} \geq \frac{f(z)-m f(y)}{z-m y} .
\end{aligned}
$$

It follows that $f$ is continuous and locally Lipschitzian in $\operatorname{int} I$.

(ii) If $0 \leq m_{1}<m_{2} \leq 1$, then every $m_{2}$-convex function is $m_{1}$-convex.

If $f:[a, b] \rightarrow \mathbb{R}$ is convex in the classical sense in the compact real interval $[a, b]$, then the values of $f$ at $a$ and $b$ can be increased without any harm for the convexity of $f$, so $f$ need not be continuous at the endpoints $a, b$. (Therefore, in the classical theory of convexity one assumes that the functions are defined on open convex sets.)

In general, the $m$-convex functions do not have this property, and it follows from the following

REMARK 3.2. Suppose that $0<m<1$ and $f$ is $m$-convex in the sense of the above definition. Then

(i) if $0 \in I$, then $f(0) \leq 0$;

(ii) if $a \in \operatorname{int} I$ and $f(a) \leq 0$, then

$$
f(x) \leq 0, \quad x \in I \cap[0, a] .
$$

Indeed, from 2.3 with $x=y=a$ we get $f((t+m(1-t)) a) \leq 0$ for all $t \in[0,1]$, so $f(x) \leq 0$ in the interval $[m a, a]$. Now, by induction, we obtain $f(x) \leq 0$ in the interval $\left[m^{n} a, a\right]$ for all $n \in \mathbb{N}$.

Hence we get the following

Corollary 3.3. Let $0<m<1$ and $0<b<+\infty$. If $f:(0, b) \rightarrow \mathbb{R}$ is $m$-convex and there is a sequence $x_{n} \in(0, b)$ such that

$$
\lim _{n \rightarrow+\infty} x_{n}=b ; \quad f\left(x_{n}\right) \leq 0 \quad \text { for all } n \in \mathbb{N},
$$

then

$$
f(x) \leq 0, \quad x \in(0, b)
$$


This feature is not shared by the classical convex functions, as they have, important in different applications, the "modality" property.

In the sequel, we assume that $I=(0,+\infty)$.

To show that there are common properties of convex functions and $m$ convex functions, we prove the following

REMARK 3.4. Let $0<m<1$. If a function $f:(0,+\infty) \rightarrow \mathbb{R}$ is $m$-affine, then there are $a, b \in \mathbb{R}$ such that

$$
f(x)=a x+b, \quad x>0 .
$$

Proof. Assume that $f$ is $m$-affine, so

$$
f(t x+m(1-t) y)=t f(x)+m(1-t) f(y), \quad x, y \in(0,+\infty), t \in[0,1]
$$

Taking arbitrarily fixed $x, y \in(0,+\infty), y<x$, and setting here

$$
z=t x+m(1-t) y, \quad t \in[0,1]
$$

we get

$$
f(z)=a z+b, \quad z \in[m y, x]
$$

where

$$
a:=\frac{f(x)-m f(y)}{x-m y}, \quad b:=m \frac{x f(y)-y f(x)}{x-m y} .
$$

Since $x$ and $y$ can be chosen arbitrarily, it follows that

$$
f(z)=a z+b, \quad z>0
$$

This property is shared by the classical convex functions.

It is well known that a real function $f$ defined in an open interval $I$ is convex iff at every point $x_{0} \in I$, the graph of $f$ is located above a supporting straight-line passing by the point $\left(x_{0}, f\left(x_{0}\right)\right)$.

The following example shows that this property is not shared by $m$-convex functions. 
Example 3.5. Let $a \in(0,+\infty)$ and $b \in\left(0, \frac{a}{2}\right)$ be two fixed real numbers. Then, the polynomial function $h: \mathbb{R} \rightarrow \mathbb{R}$ defined by

$$
h(x):=x^{4}-4(a+b) x^{3}+6 a(a+2 b) x^{2}+a^{2}(11 a+16 b) x
$$

has the following properties: its real roots are $-a$ and 0 ; its complex roots are

$$
\frac{1}{2}\left(5 a+4 b \pm i \sqrt{19 a^{2}+24 a b-16 b^{2}}\right)
$$

$h([0,+\infty))=[0,+\infty) ; f:=\left.h\right|_{[0,+\infty)}$ is strictly increasing and not convex on $[0,+\infty) ; f$ is $m$-convex for

$$
m \leq m(a, b)=\frac{(a-2 b)(a+2 b)^{3}}{(a-b)^{2}\left(a^{2}+6 a b+11 b^{2}\right)} .
$$

Proof. Since

$$
h(x)=x(x+a)\left(x^{2}-(5 a+4 b) x+11 a^{2}+16 a b\right),
$$

$-a$ and 0 are roots of $h$. In turn, the quadratic polynomial given above has discriminant

$$
\Delta=[-(5 a+4 b)]^{2}-4\left(11 a^{2}+16 a b\right)=-19 a^{2}-24 a b+16 b^{2}
$$

and $\Delta<0$ if and only if $b \in\left(\frac{3-2 \sqrt{7}}{4} a, \frac{3+2 \sqrt{7}}{4} a\right)$. But, by hypothesis, $b$ belongs to $\left(0, \frac{a}{2}\right)$ which is a proper subset of $\left(\frac{3-2 \sqrt{7}}{4} a, \frac{3+2 \sqrt{7}}{4} a\right)$. So, the other roots of $h$ are complex and they are of the indicated form.

The next property follows from the facts that $h$ is continuous,

$$
\lim _{x \rightarrow+\infty} h(x)=+\infty
$$

and its only root in $[0,+\infty)$ is 0 . Since

$$
f^{\prime \prime}(x)=12\left(x^{2}-2(a+b) x+a(a+2 b)\right)=12(x-a)(x-(a+2 b)),
$$

the function $f$ is convex in $[0, a)$ and $(a+2 b,+\infty)$ and concave in $(a, a+2 b)$. Consequently, $h$ is not convex.

Since $f$ is the product of the identity and the polynomial of degree three which is strictly increasing in $[0,+\infty)$, it is strictly increasing. 
To show the last property we apply formula (3) given in [16] with $m$ instead of $p$ denoted by $m(f)$; that is,

$$
m(f)=\inf \left\{\frac{x f^{\prime}(x)-f(x)}{y f^{\prime}(x)-f(y)}: f^{\prime \prime}(x)=0, f^{\prime}(x)=f^{\prime}(y), x, y>0\right\} .
$$

First we have to check that $x f^{\prime}(x)-f(x)>0$ for all $x \in(0,+\infty)$ (i.e., $f$ is strictly starshaped on $(0,+\infty))$. In fact,

$$
\begin{aligned}
x f^{\prime}(x)-f(x) & =3 x^{4}-8(a+b) x^{3}+6 a(a+2 b) x^{2} \\
& =3 x^{2}\left(x^{2}-\frac{8}{3}(a+b) x+2 a(a+2 b)\right) \\
& =3 x^{2}\left[\left(x-\frac{4}{3}(a+b)\right)^{2}+\frac{16}{9}\left(\frac{a}{2}-b\right)\left(\frac{a}{4}+b\right)\right]>0
\end{aligned}
$$

for all $x \in(0,+\infty)$. We already know that $f^{\prime \prime}(x)=0$ if and only if $x=a$ or $x=a+2 b$. Set $x_{1}=a$ and $x_{2}=a+2 b$. Performing a simple calculation we get

$$
f^{\prime}\left(x_{1}\right)=15 a^{3}+28 a^{2} b, \quad f^{\prime}\left(x_{2}\right)=15 a^{3}+28 a^{2} b-16 b^{3} .
$$

Solving for $y$ on each of the equations

$$
f^{\prime}(y)=15 a^{3}+28 a^{2} b, \quad f^{\prime}(y)=15 a^{3}+28 a^{2} b-16 b^{3},
$$

we get the solutions $y_{11}=a+3 b$ or $y_{12}=a$ and $y_{21}=a-b$ or $y_{22}=a+2 b$, respectively. The next step consists in evaluating the function of two variables

$$
\Phi(x, y):=\frac{x f^{\prime}(x)-f(x)}{y f^{\prime}(x)-f(y)}
$$

at four points $\left(x_{1}, y_{11}\right),\left(x_{1}, y_{12}\right),\left(x_{2}, y_{21}\right)$ and $\left(x_{2}, y_{22}\right)$. In fact,

$$
\Phi\left(x_{1}, y_{11}\right)=\frac{a^{3}(a+4 b)}{a^{4}+4 a^{3} b+27 b^{4}}, \quad \Phi\left(x_{2}, y_{21}\right)=\frac{(a-2 b)(a+2 b)^{3}}{(a-b)^{2}\left(a^{2}+6 a b+11 b^{2}\right)}
$$

and

$$
\Phi\left(x_{1}, y_{12}\right)=\Phi\left(x_{2}, y_{22}\right)=1
$$


To conclude, we have to compare all these values. Observe that all are positive. Set

$$
\begin{gathered}
A=a^{3}(a+4 b), \quad B=a^{4}+4 a^{3} b+27 b^{4}, \\
C=(a-2 b)(a+2 b)^{3}, \quad D=(a-b)^{2}\left(a^{2}+6 a b+11 b^{2}\right) .
\end{gathered}
$$

Then,

$$
\Phi\left(x_{11}, y_{11}\right)>\Phi\left(x_{2}, y_{21}\right) \Leftrightarrow A D-B C>0 .
$$

Since $A D-B C=432(a+b) b^{7}$ and $\Phi\left(x_{2}, y_{21}\right)<1$, we get

$$
m(f)=\min \left\{\Phi\left(x_{1}, y_{11}\right), \Phi\left(x_{2}, y_{21}\right), 1\right\}=\Phi\left(x_{2}, y_{21}\right),
$$

which completes the proof.

Proposition 3.6. For every $m \in(0,1)$ there is a polynomial $h$ of degree 4 such that $f:=\left.h\right|_{[0,+\infty)}$ has the following properties:

(i) $f(0)=0$;

(ii) $f$ is a diffeomorphic mapping of $[0,+\infty)$;

(iii) $f$ is $m$-convex in $[0,+\infty)$, and its epigraph $E(f)$ is an $m$-convex subset of $\mathbb{R}^{2}$;

(iv) $f$ is not convex, and its epigraph $E(f)$ is not a convex subset of $\mathbb{R}^{2}$;

(v) for any sequence $t_{n} \in(0,1), n \in \mathbb{N}$, such that

$$
\lim _{n \rightarrow+\infty} t_{n}=1
$$

there is a sequence $s_{n} \in(0,1), n \in \mathbb{N}$, such that

$$
\lim _{n \rightarrow+\infty} s_{n}=0 ; \quad t_{n}+s_{n}<1 \quad \text { for every } n \in \mathbb{N},
$$

and

$$
f\left(t_{n} x+s_{n} y\right) \leq t_{n} f(x)+s_{n} f(y), \quad x, y \in[0,+\infty), n \in \mathbb{N} .
$$


Proof. Take arbitrarily fixed $m \in(0,1), a>0$ and put $b=\frac{a}{2} r$ where $r \in[0,1]$. Then, clearly, $b \in\left[0, \frac{a}{2}\right]$ and, in view of Example 3.5, we have

$$
m(r):=m\left(a, \frac{a}{2} r\right)=\frac{16(1-r)(2+r)^{3}}{(2-r)^{2}\left(4+12 r+11 r^{2}\right)}, \quad r \in[0,1],
$$

(so $m(r)$ does not depend on $a$ ). Since $m(0)=1, m(1)=0$, and the function $m(r)$ is continuous and one-to-one in $[0,1]$, there exists a unique $r_{0} \in(0,1)$ such that $m\left(r_{0}\right)=m$. Applying the above example with $b=\frac{a}{2} r_{0}$ and Remark 2.3 we get the function $f$ having properties (i)-(iv). Property (v) follows from (iii).

\section{A result of a sandwich type}

Now we shall prove a result of a sandwich type. But first notice that

Remark 4.1. If $I$ is $(0,+\infty)$ or $[0,+\infty)$ and $f: I \rightarrow \mathbb{R}$ is $m$-convex, then

$$
f(m x) \leq m f(x), \quad x \in I .
$$

Theorem 4.2. Let I be $(0,+\infty)$ or $[0,+\infty)$, and $0<m<1$. Assume that $f: I \rightarrow \mathbb{R}$ is m-convex. Then

(i) there exists a convex function $h: I \rightarrow \mathbb{R}$ such that

$$
f(x) \leq h(x) \leq m f\left(\frac{x}{m}\right), \quad x \in I,
$$

or, equivalently,

$$
\frac{1}{m} h(m x) \leq f(x) \leq h(x), \quad x \in I .
$$

(ii) If

$$
m f(x) \leq f(m x), \quad x \in I,
$$

then

$$
f(x)=f(1) x, \quad x \in I .
$$


Proof. (i) Replacing $y$ in 2.3 by $\frac{y}{m}$ we obtain

$$
f(t x+(1-t) y) \leq t f(x)+m(1-t) f\left(\frac{y}{m}\right), \quad x, y \in I, t \in[0,1] .
$$

Hence,

$$
f(t x+(1-t) y) \leq t m f\left(\frac{x}{m}\right)+(1-t) m f\left(\frac{y}{m}\right), \quad x, y \in I, t \in[0,1],
$$

whence, setting

$$
g(x):=m f\left(\frac{x}{m}\right), \quad x \in I,
$$

we get

$$
f(t x+(1-t) y) \leq t g(x)+(1-t) g(x), \quad x, y \in I, t \in[0,1] .
$$

Applying the sandwich theorem [3] we conclude that there exists a (classical) convex function $h: I \rightarrow \mathbb{R}$ such that

$$
f(x) \leq h(x) \leq g(x), \quad x \in I,
$$

i.e., that

$$
f(x) \leq h(x) \leq m f\left(\frac{x}{m}\right), \quad x \in I .
$$

Since it is obvious that these inequalities are equivalent to

$$
\frac{1}{m} h(m x) \leq f(x) \leq h(x), \quad x \in I,
$$

the proof of (i) is complete.

(ii) In this case, by Remark 4.1, we have

$$
f(m x)=m f(x), x \in I,
$$

and,

$$
f(t x+(1-t) y) \leq t f(x)+m(1-t) f\left(\frac{y}{m}\right)=t f(x)+(1-t) f(y),
$$

which means that $f$ is convex. Moreover,

$$
f(0+)=0 .
$$


Now the convexity of $f$ implies that the function

$$
(0,+\infty) \ni x \mapsto \frac{f(x)}{x} \quad \text { is increasing. }
$$

But then for any $x, y \in I$ arbitrary with $0<x<y$,

$$
\frac{f(x)}{x} \leq \frac{f(y)}{y} .
$$

We assure $f$ is a constant function. Indeed, if this is not the case we can find $x_{1}, y_{1}$ with $0<x_{1}<y_{1}$ and positive integer $n$ such that $m^{n} y_{1}<x_{1}$, consequently

$$
\frac{f\left(m^{n} y_{1}\right)}{m^{n} y_{1}}=\frac{f\left(y_{1}\right)}{y_{1}} \leq \frac{f\left(x_{1}\right)}{x_{1}}<\frac{f\left(y_{1}\right)}{y_{1}}
$$

which is impossible.

In [12] it has been shown that an analogue of the sandwich theorem for convex functions (see [3]) is not true in the class of $m$-convex functions with $m \in(0,1)$.

EXAMPLE $4.3([12])$. Let us fix $m \in(0,1)$. For arbitrary fixed $a \in \mathbb{R}$ define the functions $f:[0,+\infty) \rightarrow \mathbb{R}$ and $g:[0,+\infty) \rightarrow \mathbb{R}$ by

$$
f(x):=a x+1, \quad g(x):=a x+\frac{1}{m} .
$$

Then, for all $x, y \in[0,+\infty)$ and $t \in[0,1]$, we have

$$
f(t x+m(1-t) y) \leq t g(x)+m(1-t) g(y),
$$

and, of course, $f(x) \leq g(x)$ for all $x \in[0,+\infty)$. However, there is no $m$-convex function $h:[0,+\infty) \rightarrow \mathbb{R}$ such that

$$
f(x) \leq h(x) \leq g(x), \quad x \geq 0 .
$$




\section{Remarks on $m$-convex functions in the case $m>1$}

In this case the class of $m$-convex functions $f:(0,+\infty) \rightarrow \mathbb{R}$ such that $f(0+) \leq 0$ is rather poor. Namely the following holds true.

Proposition 5.1. Let $m>1$ be fixed. If $f:(0,+\infty) \rightarrow \mathbb{R}$ is $m$-convex and

$$
\liminf _{x \rightarrow 0+} f(x) \leq 0,
$$

then $f$ is a linear function, i.e., $f(x)=f(1) x$ for all $x>0$.

Proof. By the assumption there is a positive decreasing sequence $\left(z_{n}\right.$ : $n \in \mathbb{N})$ such that $\lim _{n \rightarrow \infty} z_{n}=0$ and $\lim _{n \rightarrow \infty} f\left(z_{n}\right) \leq 0$. Let $\left(x_{n}: n \in \mathbb{N}\right)$ be an arbitrary positive sequence such that $\lim _{n \rightarrow \infty} x_{n}=0$. Without loss of generality, we can assume that $x_{1} \leq z_{1}$. Since $\lim _{n \rightarrow \infty} z_{n}=0$, for every $n \in \mathbb{N}$, there exist $k_{n}, l_{n} \in \mathbb{N}, k_{n}<l_{n}$, such that

$$
m z_{l_{n}} \leq x_{n} \leq z_{k_{n}}, \quad \lim _{n \rightarrow \infty} k_{n}=\infty
$$

Note that

$$
t_{n}:=\frac{x_{n}-m z_{l_{n}}}{z_{k_{n}}-m z_{l_{n}}} \in[0,1], \quad n \in \mathbb{N}
$$

and

$$
x_{n}=t_{n} z_{k_{n}}+m\left(1-t_{n}\right) z_{l_{n}}, \quad n \in \mathbb{N} .
$$

Hence, by the $m$-convexity of $f$, we have

$$
f\left(x_{n}\right)=f\left(t_{n} z_{k_{n}}+m\left(1-t_{n}\right) z_{l_{n}}\right) \leq t_{n} f\left(z_{k_{n}}\right)+m\left(1-t_{n}\right) f\left(z_{l_{n}}\right)
$$

for every $n \in \mathbb{N}$. Letting here $n \rightarrow \infty$ we get

$$
\limsup _{n \rightarrow \infty} f\left(x_{n}\right) \leq 0
$$

which proves that

$$
\limsup _{x \rightarrow 0+} f(x) \leq 0
$$


Since $m>1$, we can choose $t \in(0,1)$ such that the numbers

$$
\alpha:=t, \quad \beta:=m(1-t),
$$

fulfill the inequalities

$$
0<\alpha<1<\alpha+\beta,
$$

and $f$ satisfies the linear functional inequality

$$
f(\alpha x+\beta y) \leq \alpha f(x)+\beta f(y), \quad x, y \in(0,+\infty) .
$$

Since $\lim \sup _{x \rightarrow 0+} f(x) \leq 0$, the result follows from [11, Theorem 1] (see also [9, 10, 14]).

REMARK 5.2. If, in the proposition above, $f:[0,+\infty) \rightarrow \mathbb{R}$ is $m$-convex and $f(0)=0$, one can also apply a simple direct reasoning.

First, let us observe that, by $m$-convexity where $m>1$, there exist real numbers $\alpha, \beta$ such that $0<\alpha<1<\alpha+\beta, \frac{\log \beta}{\log \alpha}$ is an irrational number and 5.1) holds. Taking $y=x$ in 5.1), we have

$$
f((\alpha+\beta) x) \leq(\alpha+\beta) f(x), \quad x \in(0,+\infty),
$$

whence, by induction,

$$
f\left((\alpha+\beta)^{k} x\right) \leq(\alpha+\beta)^{k} f(x), \quad x \in(0,+\infty), k \in \mathbb{N} .
$$

Choose $k \in \mathbb{N}$ such that $\bar{\beta}:=\beta(\alpha+\beta)^{k}>1$. Hence, by (5.1), for all $x, y \in$ $(0,+\infty)$,

$$
\begin{aligned}
f(\alpha x+\bar{\beta} y) & =f\left(\alpha x+\beta(\alpha+\beta)^{k} y\right) \\
& \leq \alpha f(x)+\beta f\left((\alpha+\beta)^{k} y\right) \\
& \leq \alpha f(x)+\beta(\alpha+\beta)^{k} f(y)=\alpha f(x)+\bar{\beta} f(y) .
\end{aligned}
$$

So, if $\beta<1$ we can replace it by $\bar{\beta}$.

Setting $y=0$ and then $x=0$, yields

$$
f(\alpha x) \leq \alpha f(x), \quad f(\beta x) \leq \beta f(x), \quad x \in(0,+\infty),
$$


that is, $f$ satisfies the simultaneous system of two inequalities. Hence, by induction, we obtain

$$
f\left(\alpha^{k} x\right) \leq \alpha^{k} f(x), \quad f\left(\beta^{n} x\right) \leq \beta^{n} f(x), \quad x \in(0,+\infty), k, n \in \mathbb{N},
$$

whence

$$
f\left(\alpha^{k} \beta^{n} x\right) \leq \alpha^{k} \beta^{n} f(x), \quad x \in(0,+\infty), k, n \in \mathbb{N} .
$$

Now, by the continuity of $f$ in $(0,+\infty)$ (see Remark $3.1(\mathrm{i}))$ and the Kronecker theorem on the density of the set $\left\{\alpha^{k} \beta^{n}: k, n \in \mathbb{N}\right\}$, one gets

$$
f(r x) \leq r f(x), \quad r, x>0 .
$$

Replacing here $x$ by $\frac{x}{r}$ we hence get $\frac{1}{r} f(x) \leq f\left(\frac{1}{r} x\right)$ for all $r, x>0$, whence

$$
r f(x) \leq f(r x), \quad r, x>0,
$$

and, consequently,

$$
f(r x)=r f(x), \quad r, x>0 .
$$

Taking here $x=1$ we get $f(r)=f(1) r$ for all $r>0$, which completes the proof.

From Proposition 5.1 we immediately get the following

Corollary 5.3. Let $\alpha:[0,1] \rightarrow[0,1]$ and $m$ (in Definition 2.2) be such that for some $t \in(0,1)$,

$$
\min \{t, m \alpha(t)\}<1<t+m \alpha(t) .
$$

If $f:(0,+\infty) \rightarrow \mathbb{R}$ is $m$-convex wrt $\alpha$, and $\liminf _{x \rightarrow 0+} f(x) \leq 0$, then $f(x)=f(1) x$ for all $x>0$.

REMARK 5.4. In this corollary we need not to assume that a function $\alpha$ is continuous as we do in Proposition 2.1 (ii).

It follows that considering the functions which are $m$-convex wrt $\alpha$, it is rational to assume that either $t+\alpha(t) \leq 1$ for all $t \in[0,1]$ or $t+\alpha(t) \geq 1$ for all $t \in[0,1]$.

Acknowledgement. The authors are indebted to the Reviewers for their valuable remarks. 


\section{References}

[1] Aczél J., Ng C.T., A lemma on the angles between a fixed line and the lines connecting a fixed point on it with the points of a convex arc, Internat. Ser. Numer. Math. 103 (1992), 463-464.

[2] Aumann G., Konvexe Funktionen und Induktion bei Ungleichungen zwischen Mittelverten, S.-B. math.-naturw. Abt. Bayer. Akad. Wiss. München 1933, pp. 405-413.

[3] Baron K., Matkowski J., Nikodem K., A sandwich with convexity, Math. Pannonica 5 (1994), no. 1, 139-144.

[4] Daróczy Z., Páles Zs., Convexity with given infinite weight sequences, Stochastica 11 (1987), 5-12.

[5] Dragomir S.S., Toader G.H., Some inequalities for $m$-convex functions, Studia Univ. Babes-Bolyai Math. 38 (1993), no. 1, 21-28.

[6] Kuczma M., An introduction to the theory of functional equations and inequalities, Cauchy's equation and Jensen's inequality, Państwowe Wydawnictwo Naukowe and Uniwersytet Śląski, Warszawa-Kraków-Katowice, 1985; Second edition (edited by A. Gilányi), Birkhäuser, Basel, 2008.

[7] Lara T., Sánchez J.L., Rosales E., New properties of $m$-convex functions, Internat. J. Math. Anal. 9 (2015), no. 15, 735-742.

[8] Lara T., Merentes N., Quintero R., Rosales E., On strongly m-convex functions, Math. Aeterna 5 (2015), no. 3, 521-535.

[9] Matkowski J., A functional inequality characterizing convex functions, conjugacy and a generalization of Hölder's and Minkowski's inequalities, Aequationes Math. 40 (1990), $169-180$.

[10] Matkowski J., L $L^{p}$-like paranorms, in: Selected topics in functional equations and iteration theory, Proceedings of the Austrian-Polish Seminar, Universität Graz, October 24-26, 1991 (edited by D. Gronau, L. Reich), Grazer Math. Ber., 316, Karl-FranzensUniv. Graz, Graz, 1992, pp. 103-139.

[11] Matkowski J., Pycia M., Convex-like inequality, homogeneity, subadditivity, and a characterization of $L^{p}$-norm, Ann. Polon. Math. 60 (1995), 221-230.

[12] Matkowski J., Wróbel M., Remark on m-convexity and sandwich theorem, J. Math. Anal. Appl. 451 (2017), 924-930.

[13] Mocanu P.T., Serb I., Toader G.H., Real star-convex functions, Studia Univ. BabesBolyai Math. 42 (1997), no. 3, 65-80.

[14] Pycia M., Linear functional inequalities - a general theory and new special cases, Dissertationes Math. 438 (2006), 62 pp.

[15] Toader G.H., Some generalizations of the convexity, Proc. Colloq. Approx. Optim. Cluj-Napoca (Romania) 1984, pp. 329-338.

[16] Toader S., The order of a star-convex function, Bull. Appl. Comp. Math. 85 (1998), $347-350$.

TEODORO LARA

Departamento de Física y Matemáticas

UniVERSIDAd DE LOS ANDES

N. U. RAFAEL RANGEL

Trujillo

Venezuela

e-mail: tlara@ula.ve
Janusz MatKowski

Faculty of Mathematics,

Computer Science and Econometrics University of Zielona Góra

SZAFrana $4 \mathrm{~A}$

65-516 Zielona Góra

POLAND

e-mail: J.Matkowski@wmie.uz.zgora.pl 
Nelson Merentes

Escuela de Matemáticas

Universidad Central de Venezuela

CARACAS

Venezuela

e-mail: nmerucv@gmail.com
Roy Quintero

Departamento de Física y Matemáticas

Universidad de los ANDES

N. U. RAFAel RANGel

Trujillo

Venezuela

e-mail: rquinter@ula.ve

Matgorzata Wróbel

Institute of Mathematics

Czestochowa University of Technology

al. Armit Krajowej 21

42-200 Częstochowa

POLAND

e-mail: malgorzata.wrobel@im.pcz.pl 